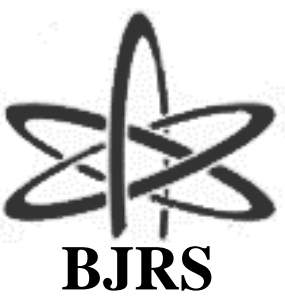

BRAZILIAN JOURNAL

$\mathrm{OF}$

RADIATION SCIENCES

08-03B (2021) 01-15

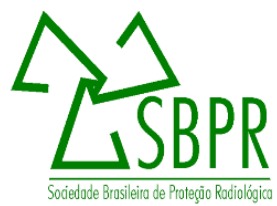

\title{
Solution of the Multigroup Neutron Diffusion \\ Eigenvalue Problem in Slab Geometry by Modified Power Method
}

\author{
R. Zanette ${ }^{\mathrm{a}}$; C. Z. Petersen ${ }^{\mathrm{b}}$; M. G. Tavares ${ }^{\mathrm{b}}$ \\ ${ }^{a}$ Programa de Pós-graduação em Matemática Aplicada, Universidade Federal do Rio Grande do Sul, \\ Av. Bento Gonçalves, 9500, 91509-900 Porto Alegre, RS, Brazil \\ rodrigozanette@hotmail.com \\ ${ }^{b}$ Programa de Pós-graduação em Modelagem Matemática, Universidade Federal de Pelotas, \\ Campus Universitário, s/n, 96160-000 Capão do Leão, RS, Brazil. \\ claudiopetersen@yahoo.com.br, matheus.gulartetavares@gmail.com
}

\begin{abstract}
We describe in this work the application of the modified power method for solving the multigroup neutron diffusion eigenvalue problem in slab geometry considering two-dimensions for criticality global calculations. In order to solve this problem a modified power method is used to obtain the dominant eigenvalue (effective multiplication factor) and its corresponding eigenfunction (scalar neutron flux). The innovation of this work is solving the neutron diffusion equation in analytical form for each new iteration of the power method. For solve this problem we propose to apply the Finite Fourier Sine Transform on one of the variables obtaining a transformed problem which is resolved by well-established methods for ordinary differential equations. The inverse Fourier Transform is used to reconstruct the solution for the original problem. In order to maintain the analytical character for the solution in each new iteration of the power method the neutron flux is reconstructed through a polynomial interpolation. The methodology is implemented to solve a homogeneous and heterogeneous problems and the results are compared with works presents in the literature.
\end{abstract}

Keywords: neutron diffusion equation, eigenvalue problem, modified power method, polynomial interpolation. 


\section{INTRODUCTION}

The neutron population distribution in a nuclear reactor varies according to its rate of neutron production and its rate of neutron losses. When the production of neutrons exceeds the losses it is said that the reactor is in the supercritical state. When the neutron population remains steady with successive generations, it is referred to as a critical state and when losses dominate the production, it is considered subcritical state.

The criticality of a nuclear reactor is obtained by solving the neutron diffusion eigenvalue problem. The solution of this problem, in addition, to provide the critical eigenvalue, provides the neutron flux distribution and the fractional power by region.

One of the first methods used for solving this eigenvalue problem was the finite difference method (FDM), however as it requires very mesh points to model accurately, it becomes a bit slow from the computational point of view [1]. Over time other methodologies have been implemented for solving the neutron diffusion eigenvalue problem, as for example: Finite Element Method [2], [Pseudo-Harmonics Expansion Method [1], Finite Volume Method [3], Lagrange Polynomial Algorithms (LAP) using the same approach in FDM [4], Taylor Series Expansion Method [5], Isogeometric Analysis [6], Integral Transform Techniques [7] and Generalized Integral Transform Technique (GITT) combined with Laplace Transform given by [8] and Nodal Method [9].

In order to develop method that can solve the eigenvalue problem and predict the behavior of the neutron flux in the nuclear reactor core with accuracy and reliability, this work proposes to apply the modified power method in the multigroup neutron diffusion eigenvalue problem in slab geometry considering two spatial dimensions. The main idea is to solve the neutron diffusion equation in analytical form for each iteration of the power method. As the equations are partial differential equations applies the Finite Fourier Sine Transform in one of the spatial dimensions falling back on a transformed problem decoupled by the power method. The transformed problem is solved by well-established methods for ordinary differential equations. For the neutron flux expression always remain in a standard form for all iterations, it is proposed that the expression be reconstructed through a polynomial interpolation. 


\section{MATHEMATICAL FORMULATION}

\subsection{The neutron diffusion equation}

The steady state multigroup neutron diffusion equation considering homogeneous nuclear parameters can be written as [9]:

$$
-D_{g} \nabla^{2} \phi_{g}+\Sigma_{R g} \phi_{g}=\frac{1}{K_{e f f}} \chi_{g} \sum_{g^{\prime}=1}^{G} \nu_{g^{\prime}} \Sigma_{f g^{\prime}} \phi_{g^{\prime}}+\sum_{g^{\prime}=1 ; g^{\prime} \neq g}^{G} \Sigma_{s g^{\prime} g} \phi_{g^{\prime}},
$$

where

$g=1, \ldots, G$ are neutron energy groups; $D_{\mathrm{g}}$ is the diffusion coefficient of the energy group $g ; \phi_{g}$ is the neutron scalar flux of the energy group $g ; \Sigma_{R g}$ is the removal cross section of the energy group $\mathrm{g} ; K_{\text {eff }}$ is the effective multiplication factor; $\chi_{g}$ is the integrated fission spectrum of the energy group $g, v_{g}$ is the average number of neutrons emitted by fission of the energy group $g ; \Sigma_{f g}$ is the fission cross section of the energy group $g ; \Sigma_{s g^{\prime} g}$ is the scattering cross section from energy group $g^{\prime}$ to $g$.

In this work we consider a rectangular geometry in which the most usual boundary conditions for the system of equations (1) can be written in the form:

$$
\alpha \phi_{g}+\beta \frac{\partial \phi_{g}}{\partial n}=0
$$

where $\alpha$ and $\beta$ are constants with $|\alpha|+|\beta|>0$ and $n$ depends on the contour.

Without loss of generality, the above equation for two dimensions in Cartesian geometry and constants nuclear parameters by region becomes:

$-D_{g}^{(r)}\left(\frac{\partial^{2} \phi_{g}^{(r)}(x, y)}{\partial x^{2}}+\frac{\partial^{2} \phi_{g}^{(r)}(x, y)}{\partial y^{2}}\right)+\Sigma_{R g}^{(r)} \phi_{g}^{(r)}(x, y)=\frac{\chi_{g}}{K_{e f f}} \sum_{g^{\prime}=1}^{G} v_{g^{\prime}} \Sigma_{f g^{\prime}}^{(r)} \phi_{g^{\prime}}^{(r)}(x, y)+\sum_{\substack{g^{\prime}=1 \\ g^{\prime} \neq g}}^{G} \Sigma_{s g^{\prime}}^{(r)} \phi_{g^{\prime}}^{(r)}(x, y)$,

where $r=1, \ldots, R$, with $r$ belonging to domain $\Gamma$ and contour $\partial \Gamma_{x y}$ satisfying $0 \leq x \leq L_{1}$ and $0 \leq y \leq L_{2}, D_{g}^{(r)}$ is the diffusion coefficient of the energy group $g$ in the region $r ; \phi_{g}^{(r)}(x, y)$ is the 
neutron scalar flux of the energy group $g$ in the region $r ; \Sigma_{R g}^{(r)}$ is the removal cross section of the energy group $\mathrm{g}$ in the region $r ; \Sigma_{f g^{\prime}}^{(r)}$ is the fission cross section of the energy group $g^{\prime}$ in the region $r ; \Sigma_{s g^{\prime} g}^{(r)}$ is the scattering cross section from energy group $g^{\prime}$ to $g$ in the region $r$.

The partial differential equations above on a domain $\Gamma$ with boundary $\partial \Gamma$ satisfies the following usual boundary condition in reactor physics: zero-flux on the external borders and reflective condition in the reactor center.

In addition to the boundary conditions, there appear the piecewise open interface conditions, that combine the solutions of adjacent regions ( $r$ and $r^{\prime}$, respectively) to one unique solution of the whole problem known as flux and current continuity given by:

$$
\begin{gathered}
\left.\phi_{g}^{(r)}\right|_{\partial \Gamma_{x y}}=\left.\phi_{g}^{\left(r^{\prime}\right)}\right|_{\partial \Gamma_{x y}} \\
\left.D_{g}^{(r)} \nabla \phi_{g}^{(r)}\right|_{\partial \Gamma_{x y}}=\left.D_{g}^{\left(r^{\prime}\right)} \nabla \phi_{g}^{\left(r^{\prime}\right)}\right|_{\partial \Gamma_{x y}}
\end{gathered}
$$

For the development of this work is also considered the following simplifications:

- $\quad$ problems without scattering from lower energy to higher energy groups (up-scattering);

- two energy groups $(g=1: 2)$.

Thus, the equations (3) can be rewritten in the following matrices system by region as:

$$
\begin{gathered}
\mathbf{M} \boldsymbol{\Phi}=\frac{1}{K_{\text {eff }}} \mathbf{F} \boldsymbol{\Phi} \\
\text { where } \mathbf{M}=\left(\begin{array}{cc}
\nabla^{2}-\frac{\Sigma_{R 1}}{D_{1}} & 0 \\
\frac{\Sigma_{s 12}}{D_{2}} & \nabla^{2}-\frac{\Sigma_{R 2}}{D_{2}}
\end{array}\right), \boldsymbol{\Phi}=\left(\begin{array}{l}
\phi_{1}(x, y) \\
\phi_{2}(x, y)
\end{array}\right), \mathbf{F}=\left(\begin{array}{cc}
-\frac{v_{1} \Sigma_{f 1}}{D_{1}} & -\frac{v_{2} \Sigma_{f 2}}{D_{1}} \\
0 & 0
\end{array}\right) \text { and source term }
\end{gathered}
$$

given by: $\mathbf{S}=\mathbf{F} \boldsymbol{\Phi}$. 


\subsection{Power method}

In order to solve the eigenvalue problem, we propose to use a well-known iterative source method known power method. The basic steps this method are:

- Initial estimate for neutrons fluxes, $\phi_{1}^{[1]}(x, y), \phi_{2}^{[1]}(x, y)$ and the eigenvalue, $K_{e f f}^{[1]}$;

- Solve the equation system: $\mathbf{M} \boldsymbol{\Phi}^{[i+1]}=\frac{1}{K_{e f f}^{[i]}} \mathbf{S}^{[i]}$

- Update of the source term, $\mathbf{S}^{[i]}$;

- Update of the eigenvalue, $K_{\text {eff }}^{[i+1]}=K_{\text {eff }}^{[i]} \frac{\iint \mathbf{S}^{[i+1]} d x d y}{\iint \mathbf{S}^{[i]} d x d y}$

- Stop criterion: $\frac{\left|K_{\text {eff }}^{[i+1]}-K_{\text {eff }}^{[i]}\right|}{\left|K_{\text {eff }}^{[i+1]}\right|}<\grave{\mathrm{o}}_{1}, \frac{\left|\iint \mathbf{S}^{[i+1]}-\mathbf{S}^{[i]} d x d y\right|}{\left|\iint \mathbf{S}^{[i+1]} d x d y\right|}<\grave{\mathrm{o}}_{2}$;

- If the changes are greater than the stop criterions, then set and return at step-2, otherwise end the iterations.

This algorithm is implemented to solve the eigenvalue problem in a classic way. In the subsection following we are going to present the modified power method.

\subsection{Modified power method}

The innovation of this work is to solve the neutron diffusion equations in analytical form for each new iteration of the power method.

The diffusion equation system as it stands is unlikely to be solved exactly in closed and finite analytical form. In order to introduce a simplification which nevertheless permits to control convergence in a strict mathematical sense, one may make use of the physical resolution scale set by the inverse of the largest macroscopic cross-section value of the problem in consideration and segment the sheet into several regions $r \in\{1, \ldots, R\}$, with linear dimensions smaller than the mean free path [7].

We suppose that in a stationary regime that dependence on the specific energy group, the physical coefficients are now "locally" homogeneous, i.e. constant in a specific region $r$ 
$\left(D_{g} \rightarrow D_{g}^{r}, \Sigma_{r g} \rightarrow \Sigma_{r g}^{r}, \Sigma_{f g^{\prime}} \rightarrow \Sigma_{f g^{\prime}}^{r}, \Sigma_{g g^{\prime}} \rightarrow \Sigma_{g g^{\prime}}^{r}\right)$. The only quantity that preserves its original dependence on the coordinates is the scalar neutron flux, which is determined in its analytical form for each region $\left(\phi(x, y) \rightarrow \phi^{r}(x, y)\right)$.

Thus, it is proposed to apply the Finite Fourier Sine Transform (FFST) on one of the spatial variables obtaining transformed problem, falling into a decoupled assembly of ordinary differential equations (ODE's). This type transform (FFST) is applied because the domain is finite and as the most problems are considered null external frontiers your inverse is easily implemented.

One way to solve these equations is to consider symmetry in relation to the axes and apply an integral transform to one of the spatial dimensions, for example y, although this simplification is not always possible.

In this article we chose to apply the Fourier Transform Method. Without loss of generality, we are going to demonstrate the method with the Finite Fourier Sine Transform (FFST) $\phi=0$ boundary conditions both in $x=0$ and $x=L_{1}$ or $y=0$ and $y=L_{2}$.

The FFST of a continuous function $\phi(x, y)$ in a finite interval $0<y<L_{2}$ is defined by:

$$
\mathrm{F}_{s}\{\phi(x, y)\}=\hat{\phi}(x, n)=\int_{0}^{L_{2}} \phi(x, y) \sin \left(\frac{n \pi y}{L_{2}}\right) d y \quad \text {, }
$$

where $\hat{\phi}(x, n)$ is the transform neutron flux and $n$ is an integer. Once the FFST is applied to the $y$ coordinate, we fall into a set of second-order ordinary differential equations at $x$. When applying the Finite Fourier Sine Transform to the variable $y$ of the proposed problem, a system of ordinary differential equations (ODE's) is established. Without loss of generality, the application of (FFST) in (3) for each region considering homogeneous parameters, two energy groups, without upscattering and with zero flux in the boundary results in:

$$
\begin{aligned}
& \frac{d^{2} \hat{\phi}_{1}(x, n)}{d x^{2}}-\frac{n^{2} \pi^{2} \hat{\phi}_{1}(x, n)}{L_{2}^{2}}-\frac{\Sigma_{R 1} \hat{\phi}_{1}(x, n)}{D_{1}}=-\frac{1}{K_{e f f}}\left(\frac{v_{1} \Sigma_{f 1} \hat{\phi}_{1}(x, n)}{D_{1}}+\frac{v_{2} \Sigma_{f 2} \hat{\phi}_{2}(x, n)}{D_{1}}\right) \\
& \frac{d^{2} \hat{\phi}_{2}(x, n)}{d x^{2}}-\frac{n^{2} \pi^{2} \hat{\phi}_{2}(x, n)}{L_{2}^{2}}-\frac{\Sigma_{R 2} \hat{\phi}_{2}(x, n)}{D_{2}}=-\frac{\Sigma_{s 12} \hat{\phi}_{1}(x, n)}{D_{2}}
\end{aligned}
$$


The ODE's that arise from the application of FFST are resolved by well-established methods in the literature. After obtaining the EDO's solutions, the inverse Fourier transform is used to reconstruct the solution for the original problem. This inverse transform is given for a series:

$$
\phi_{g}(x, y)=\frac{2}{L} \sum_{n=1}^{\infty} \hat{\phi}_{g}(x, n) \sin \left(\frac{n \pi y}{L}\right),
$$

where $L$ is thickness of domain in $y$.

This sum must be truncated in a given $n$, when properly chosen, it results in small loss of information. For this, we propose as a stop criterion the sum of the module of the element that is being added as:

$$
\left\|\hat{\phi}_{g}\left(x_{i}, n\right)\right\|_{2}<\varepsilon_{3},
$$

where $x_{i}$ is the point at which the sum of the inversion is being calculated and $\varepsilon_{3}$ is a very small arbitrary constant. By inspection, it is observed that with the increase $n$ the contributions decrease, making the solution convergent in each point of the domain.

It is known, the power method is an iterative source method in which is updated by the neutron flux expression of previous iteration. With the successive iterations the flux expression becomes ever larger and more complex, since the flux is express in analytical form.

At each iteration of the power method it is necessary to update the source term with the neutron flux and the $K_{\text {eff }}$ of the previous iteration. Thus in all iterations new terms are added which becomes the process very laborious. To overcome this problem is proposed to reconstruct the neutron flux in standard form by polynomial interpolation in $x$ and $y$ and this new neutron flux updates the source term of the next iteration.

Therefore, the proposed alternative to overcome this problem and reconstruct the expression of flux, with each iteration, always in the same standard form. For this, one must evaluate to express the neutron flux in equally spaced points and then interpolates them in a polynomial of the same order in all iterations given by:

$$
\phi(x, y)=\left(\sum_{n=1}^{N} c_{n} x_{n}\right)\left(\sum_{m=1}^{M} d_{m} y_{m}\right),
$$


where $c_{n}$ and $d_{m}$ are constants and $\mathrm{N}$ and $\mathrm{M}$ represent the order of the polynomials in $\mathrm{x}$ and $\mathrm{y}$, respectively. To find the constants $c_{n}$ and $d_{m}$ in a unique way, the boundary conditions and current and flux continuity interfaces are used apply in order to establish a determined linear system.

To solve the linear system generated by the polynomial interpolation QR decomposition method was used. The choice this method is due to the fact that the number of points inside each sub region changes according to the chosen interval and QR decomposition, because in the system linear $\mathbf{A X}=$ $\mathbf{B}$, the matrix A most of the time is no square. Another aspect favorable is that the matrix $\mathrm{R}$ is a superior triangular matrix and better conditioned than the matrix $\mathbf{A}$, and $\mathbf{Q}$ and an orthonormal matrix [10].

\section{RESULTS AND DISCUSSION}

\subsection{Homogeneous Case Test}

In this section are compared the numerical results with work presented by [8]. This problem is composed for a square homogeneous region of $6 \mathrm{~cm}$ thickness, two energy groups (fast and thermal), zero-flux boundary conditions on the exterior, $\left(\phi_{\mathrm{g}}(\mathrm{x}, 6)=\phi_{\mathrm{g}}(6, \mathrm{y})=0\right)$, and reflection boundary conditions on the planes of symmetry, $\left(\frac{\partial \phi_{\mathrm{g}}(\mathrm{x}, 0)}{\partial \mathrm{x}}=\frac{\partial \phi_{\mathrm{g}}(0, \mathrm{y})}{\partial \mathrm{y}}=0\right)$. The remaining parameters are given in Table 1.

Table 1: Nuclear parameters for the homogeneous problem.

\begin{tabular}{ccccc}
\hline Group & $\mathrm{D}_{\mathrm{g}}$ & $\Sigma_{\mathrm{Rg}}$ & $v \Sigma_{\mathrm{fg}}$ & $\Sigma_{\mathrm{sg}{ }^{\prime}}$ \\
\hline 1 & 1.6497 & 0.02309 & 0.005008 & 0.00000 \\
2 & 0.4754 & 0.07886 & 0.09713 & 0.01423 \\
\hline
\end{tabular}

For use the Finite Fourier Sine Transform is needed that the boundaries be all null and the domain be non-negative. Since boundary conditions are reflective at zero, thus it is proposed to expand the problem for others quadrants displaced to the right and up as Figure 1. 
Besides that in the proposed method, the neutron flux must be interpolated in a standard form in all iterations. In this problem is used the following interpolation:

$$
\phi_{\mathrm{g}}=\left(\mathrm{c}_{1}+\mathrm{c}_{2} \mathrm{x}+\mathrm{c}_{3} \mathrm{x}^{2}+\mathrm{c}_{4} \mathrm{x}^{3}\right)\left(\mathrm{c}_{5}+\mathrm{c}_{6} \mathrm{y}+\mathrm{c}_{7} \mathrm{y}^{2}+\mathrm{c}_{8} \mathrm{y}^{3}+\mathrm{c}_{9} \mathrm{y}^{4}\right) .
$$

Figure 1: Domain adapted to solve the problem.
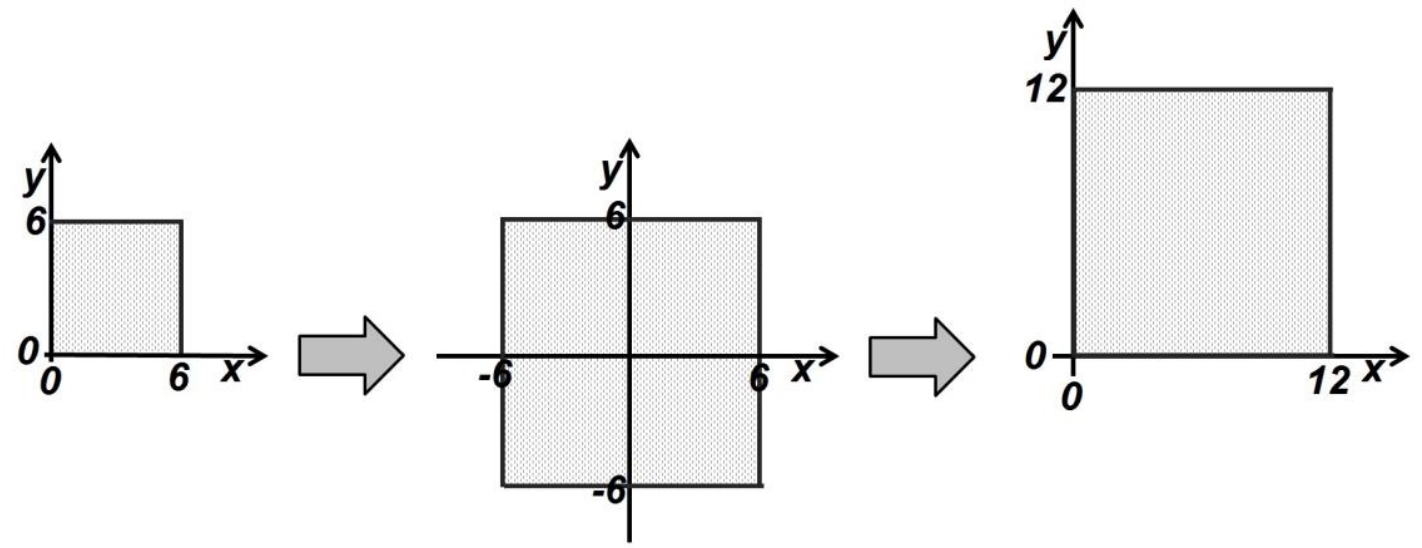

This form given by (11) is the best combination of agreement of the results and computational efficiency to get the $K_{\text {eff }}=0.0585997$, while the value obtained by [8] is $K_{\text {eff }}^{r e f}=0.0585989$. It can be observed a precision of 5 digits with the reference adopted, which suggests an excellent agreement for global calculations in reactor physics. It is remembering that the domain has been expanded and dislocate, thus, after obtain solution, it must turn back to its original position.

In the Table 2 are presented the normalized fast neutron flux, these are compared with those obtained by [8] represented by subscript "ref". In addition, we calculate the relative error with respect to the reference as:

$$
\text { Relative Error }=\left|\frac{\phi^{r e f}(x, y)-\phi(x, y)}{\phi^{r e f}(x, y)}\right| .
$$


Table 2: Comparation of the quantitative results of fast neutron flux.

\begin{tabular}{ccccccc}
\hline$y$ & $\phi_{1}(0, y)$ & $\phi_{1}^{\text {ref }}(0, y)$ & $\begin{array}{c}\text { Rel. } \\
\text { error }\end{array}$ & $\phi_{1}(3, y)$ & $\phi_{1}^{\text {ref }}(3, y)$ & $\begin{array}{c}\text { Rel. } \\
\text { error }\end{array}$ \\
\hline 0.0 & $\mathbf{1 . 0 0 0 0 0 0 0}$ & 1.00000 & 0.000000 & $\mathbf{0 . 7 0 7 1 0 6 8}$ & 0.70710 & $9.617 \mathrm{e}-06$ \\
1.5 & $\mathbf{0 . 9 2 3 8 7 9 5}$ & 0.92388 & $5.412 \mathrm{e}-07$ & $\mathbf{0 . 6 5 3 2 8 1 5}$ & 0.65327 & $1.760 \mathrm{e}-05$ \\
3.0 & $\mathbf{0 . 7 0 7 1 0 6 8}$ & 0.70711 & $4.525 \mathrm{e}-06$ & $\mathbf{0 . 5 0 0 0 0 0 0}$ & 0.49999 & $2.000 \mathrm{e}-05$ \\
4.5 & $\mathbf{0 . 3 8 2 6 8 3 4}$ & 0.38268 & $8.885 \mathrm{e}-06$ & $\mathbf{0 . 2 7 0 5 9 8 0}$ & 0.27388 & $1.198 \mathrm{e}-02$ \\
6.0 & $\mathbf{0 . 0 0 0 0 0 0 0}$ & $6.1 \mathrm{e}-17$ & - & $\mathbf{0 . 0 0 0 0 0 0 0}$ & $6.3 \mathrm{e}-17$ & - \\
\hline
\end{tabular}

Moreover, in Fig. 2 and Fig. 3 are plotted the graphs 2D and 3D for the fast and thermal neutron flux, respectively.

Figure 2: The fast and thermal neutron scalar flux at $y=0$.

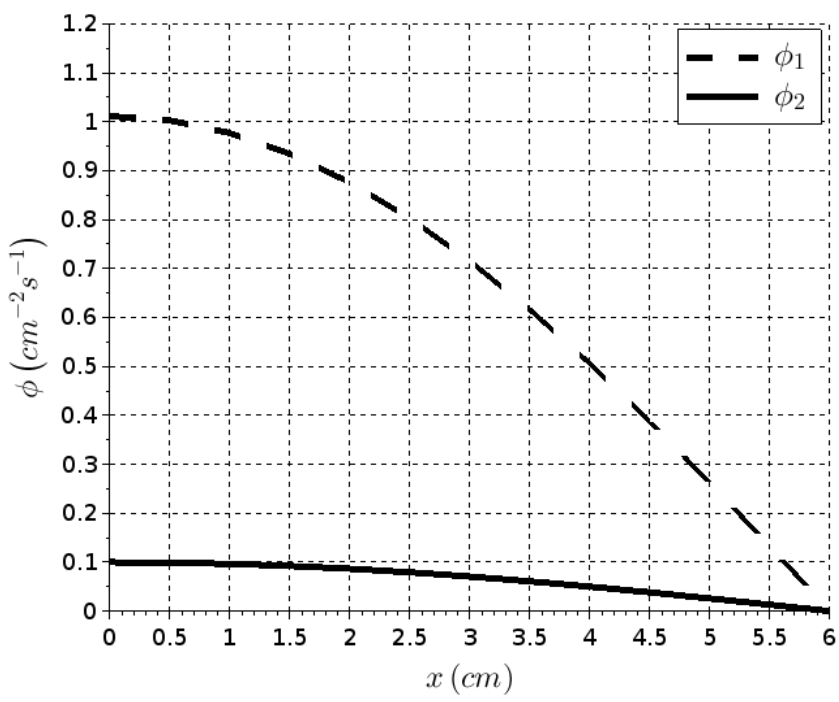


Figure 3: Fast and thermal neutron flux distributions along the domain.

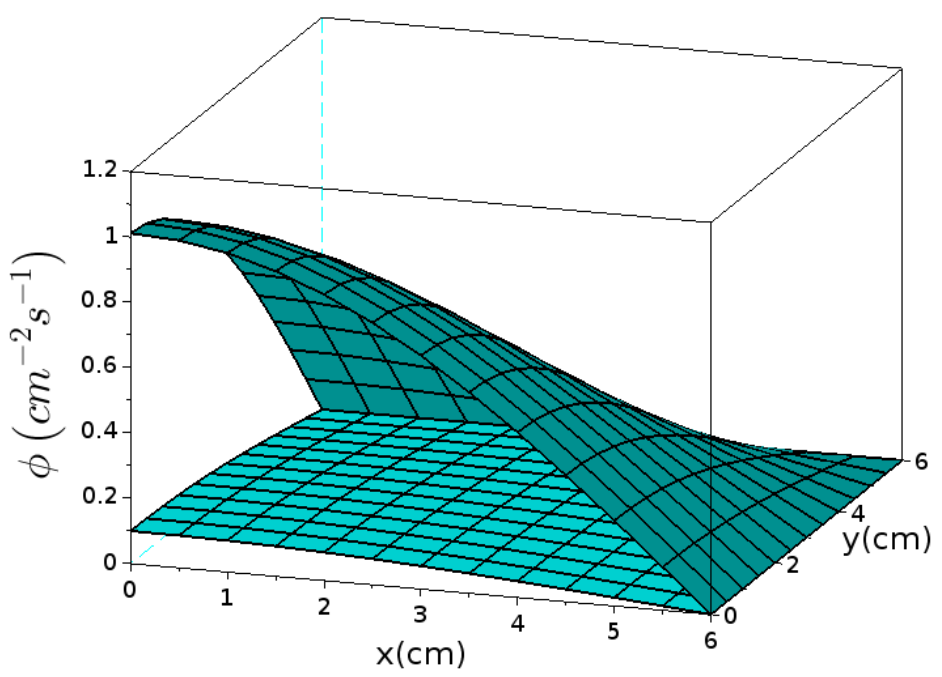

\subsection{Heterogeneous Case Test}

This problem is composed of a heterogeneous medium with two regions and two energy groups (fast and thermal), where Region 1 is a square with measures $(0 \leq x \leq 10 \mathrm{~cm}$ and $0 \leq y \leq 10 \mathrm{~cm})$ and Region 2 is a square with measures $(10 \mathrm{~cm} \leq x \leq 20 \mathrm{~cm}$ and $0 \leq y \leq 10 \mathrm{~cm})$. For both regions are considered zero-flux boundary conditions on all contours. The parameters are based on the reference [11] and presented in the Table 3.

Table 3: Nuclear parameters for the heterogeneous problem.

\begin{tabular}{cccccc}
\hline Mat. & Group & $\mathrm{D}_{\mathrm{g}}$ & $\Sigma_{\mathrm{Rg}}$ & $v \Sigma_{\mathrm{fg}}$ & $\Sigma_{\mathrm{sg}^{\prime} \mathrm{g}}$ \\
\hline 1 & 1 & 1.4 & 0.020 & 0.007 & 0.00 \\
1 & 2 & 0.4 & 0.150 & 0.200 & 0.01 \\
2 & 1 & 1.3 & 0.018 & 0.003 & 0.00 \\
2 & 2 & 0.5 & 0.050 & 0.060 & 0.01 \\
\hline
\end{tabular}


Similarly, to the homogeneous case, the form given by (11) is the best combination of agreement of the results and computational efficiency to get the $K_{\text {eff }}=0.075303$, while the value obtained by the classic nodal method developed in the same spirit of [11] is $K_{e f f}^{r e f}=0.075284$. It can be observed a precision of 3 digits with the reference adopted, which suggests a good agreement for global calculations in nuclear reactor physics. In the Table 4 and Table 5 are presented the thermal and fast neutron flux. These are compared with those obtained by classic nodal method represented by subscript "ref". In addition, we calculate the relative error with respect to the reference as Eq. (12).

It is worth noting that since the boundary conditions are null across the border, it is not necessary to adapt the contours for the application of the FFST.

Table 4: Comparation of the quantitative results of fast neutrons fluxes.

\begin{tabular}{ccccccc}
\hline $\mathbf{y}$ & $\phi_{1}(5, y)$ & $\phi_{1}^{\text {ref }}(5, y)$ & $\begin{array}{c}\text { Rel. } \\
\text { error }\end{array}$ & $\phi_{1}(15, y)$ & $\phi_{1}^{\text {ref }}(15, y)$ & $\begin{array}{c}\text { Rel. } \\
\text { error }\end{array}$ \\
\hline 0.5 & 0.1564345 & 0.1546627 & $1.146 \mathrm{e}-02$ & 0.0557265 & 0.0568993 & $2.061 \mathrm{e}-02$ \\
1.5 & 0.4539905 & 0.4488486 & $1.146 \mathrm{e}-02$ & 0.1617245 & 0.1651281 & $2.061 \mathrm{e}-02$ \\
2.5 & 0.7071068 & 0.6990981 & $1.146 \mathrm{e}-02$ & 0.2518918 & 0.2571930 & $2.061 \mathrm{e}-02$ \\
3.5 & 0.8910065 & 0.8809150 & $1.146 \mathrm{e}-02$ & 0.3174022 & 0.3240821 & $2.061 \mathrm{e}-02$ \\
4.5 & 0.9876883 & 0.9765018 & $1.146 \mathrm{e}-02$ & 0.3518431 & 0.3592477 & $2.061 \mathrm{e}-02$ \\
\hline
\end{tabular}

Table 5: Comparation of the quantitative results of thermal neutrons fluxes.

\begin{tabular}{ccccccc}
\hline $\mathbf{y}$ & $\phi_{2}(5, y)$ & $\phi_{2}^{\text {ref }}(5, y)$ & $\begin{array}{c}\text { Rel. } \\
\text { error }\end{array}$ & $\phi_{2}(15, y)$ & $\phi_{2}^{\text {ref }}(15, y)$ & $\begin{array}{c}\text { Rel. } \\
\text { error }\end{array}$ \\
\hline 0.5 & 0.0075949 & 0.0075057 & $1.188 \mathrm{e}-02$ & 0.0055640 & 0.005676 & $1.973 \mathrm{e}-02$ \\
1.5 & 0.0220414 & 0.0217823 & $1.189 \mathrm{e}-02$ & 0.0161474 & 0.0164723 & $1.972 \mathrm{e}-02$ \\
2.5 & 0.0343303 & 0.0339267 & $1.190 \mathrm{e}-02$ & 0.0251502 & 0.0256562 & $1.972 \mathrm{e}-02$ \\
3.5 & 0.0432586 & 0.0427501 & $1.189 \mathrm{e}-02$ & 0.0316911 & 0.0323287 & $1.972 \mathrm{e}-02$ \\
4.5 & 0.0479526 & 0.0473889 & $1.190 \mathrm{e}-02$ & 0.0351298 & 0.0358366 & $1.972 \mathrm{e}-02$ \\
\hline
\end{tabular}


It can be observed for both fast and thermal neutron flux a relative error in the order of $10^{-2}$ for the points considered in the problem, which is good accuracy in nuclear reactor physics. For illustration the thermal and fast neutron flux 3D graph is shown in Figures 4 and 5.

Figure 4: Fast neutron flux distributions along the domain.

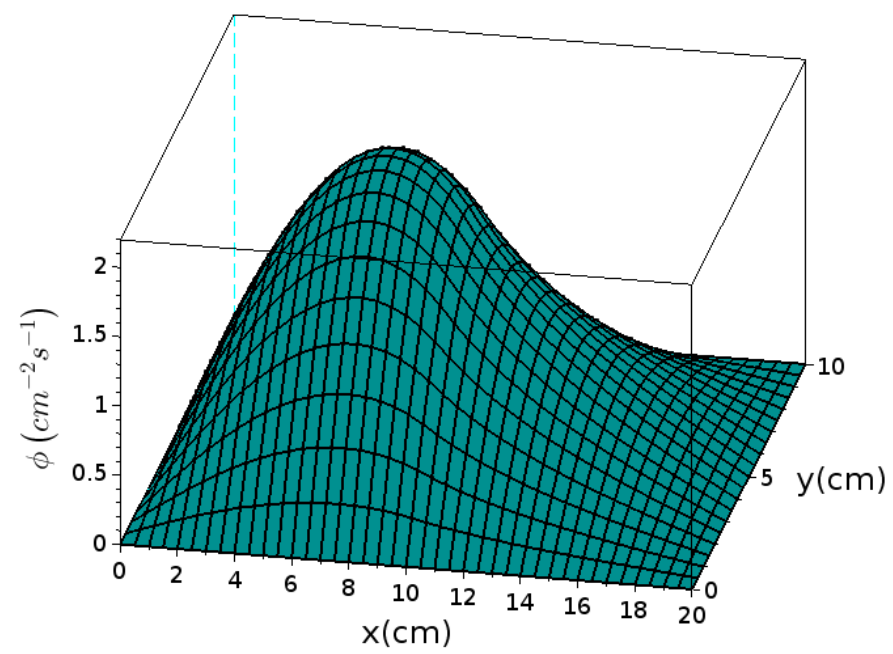

Figure 5: Thermal neutron flux distributions along the domain.

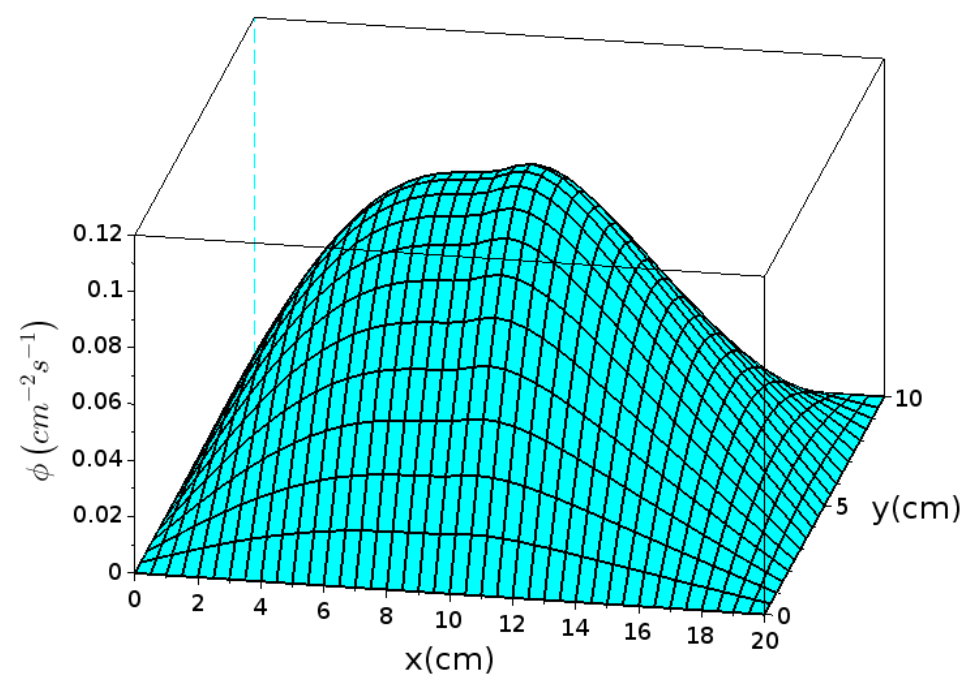




\section{CONCLUSION}

We describe in this paper a modified Power Method to solve the multigroup neutron diffusion eigenvalue problem in slab geometry considering two-dimensions. The proposed methodology was successfully developed to calculate the criticality of a nuclear reactor and obtaining the behavior of the neutron flux. The main contribution of this work relies on analytical character of the solution by Finite Fourier Sine Transform in each iteration of the known Power Method.

This modification of the Power Method provided excellent results when compared with one presented in the literature. It should be noted that the interpolation in each iteration has a standard form that provides the best result and can be adjusted for different configurations and neutron flux distribution.

In the future we intend to extend this methodology for more complex problems, for example, multidimensional multilayer space kinetics problems. We are now working in these directions and we plan to report the results after they are fully tested.

\section{ACKNOWLEDGMENT}

The authors are grateful indebted to Coordenação de Aperfeiçoamento de Pessoal de Nível Superior (CAPES) Brazil for the financial support.

\section{REFERENCES}

[1] LIMA, Z. R.; SILVA, F. C.; ALVIM, A. C. M. Solution of the fixed source neutron diffusion equation by using the pseudo-harmonics method. Annals of Nuclear Energy, v. 31, p. 1649$1666,2004$.

[2] MISFELDT, I. Solution of the Multigroup Neutron Diffusion Equations by the Finite Element Method. Danish Atomic Energy Commission, Denmark, 1975.

[3] BERNAL, A.; MIRÒ, R.; GINESTAR, D.; VERDÙ, G. Resolution of the Generalized Eigenvalue Problem in the Neutron Diffusion Equation Discretized by the Finite Volume Method. Hindawi Publishing Corporation, pp. 15, 2014. 
[4] QUINTERO-LEYVA, B. Solving the static-neutron diffusion equation in 2D-Cartesian geometry with Lagrange interpolation. Annals of Nuclear Energy, v. 65, p. 370-375, 2014.

[5] CEOLIN, C.; SCHRAMM, M.; VILHENA, M.T.; BODMANN, B.E. On the neutron multigroup kinetic diffusion equation in a heterogeneous slab: an exact solution on a finite set of discrete points. Ann. Nucl. Energy, v. 76, p. 271-282, 2015.

[6] WELCH, J.A.; KOPHAZI, J.; OWENS, A.R.; EATON, M.D. Isogeometric analysis for the multigroup neutron diffusion equation with applications in reactor physics. Ann. Nucl. Energy, V. 101, p. 465-480, 2017.

[7] BODMANN B.; VILHENA M.; FERREIRA L.; BARDAJI J. An analytical solver for the multigroup two-dimensional neutron-diffusion equation by integral transform techniques. Il Nuovo Cimento, v. 33, p. 63-70, 2010.

[8] HEINEN, I. R. Soluções Analíticas da Equação de Difusão de Nêutrons geral pelas Técnicas das Transformadas Integrais. PhD Thesis, UFRGS, Porto Alegre, Brazil, 2009.

[9] SMITH, K. An analytic nodal method for solving the two-group, multidimensional, static and transient neutron diffusion equations. $\mathrm{PhD}$ Thesis, Massachusetts Institute of Technology, Department of Nuclear Engineering, Cambridge, United States, 1979.

[10] DUDERStADT, J.; HAMILTON, J. Nuclear Reactor Analysis. New York: John Wiley \& Sons, 1976.

[11] BURDEN R. L.; FAIRES J. D. Numerical Analysis, $10^{\text {th }}$ ed: Boston: Cengage, 2016. 